\title{
Axially Symmetric Null Dust Space-Time, Naked Singularity, and Cosmic Time Machine
}

\author{
Faizuddin Ahmed \\ Hindustani Kendriya Vidyalaya, Dinesh Ojha Road, Guwahati 781005, India \\ Correspondence should be addressed to Faizuddin Ahmed; faizuddinahmed15@gmail.com
}

Received 5 April 2017; Accepted 14 May 2017; Published 12 June 2017

Academic Editor: Torsten Asselmeyer-Maluga

Copyright (C) 2017 Faizuddin Ahmed. This is an open access article distributed under the Creative Commons Attribution License, which permits unrestricted use, distribution, and reproduction in any medium, provided the original work is properly cited. The publication of this article was funded by SCOAP

We present a gravitational collapse null dust solution of the Einstein field equations. The space-time is regular everywhere except on the symmetry axis where it possesses a naked curvature singularity and admits one parameter isometry group, a generator of axial symmetry along the cylinder which has closed orbits. The space-time admits closed timelike curves (CTCs) which develop at some particular moment in a causally well-behaved manner and may represent a Cosmic Time Machine. The radial geodesics near the singularity and the gravitational lensing (GL) will be discussed. The physical interpretation of this solution, based on the study of the equation of the geodesic deviation, will be presented. It was demonstrated that this solution depends on the local gravitational field consisting of two components with amplitudes $\Psi_{2}$ and $\Psi_{4}$.

\section{Introduction}

For algebraically special metrics, the Petrov classification is a way to characterize the space-time by the number of times a principal null direction (PND) admits. The various algebraically special Petrov types have some interesting physical interpretations in the context of gravitational radiation. In the Newmann-Penrose notations $[1,2]$ (null tetrad $(\mathbf{k}, \mathbf{l}, \mathbf{m}, \overline{\mathbf{m}})$, where $\mathbf{k}, \mathbf{l}$ are real and $\mathbf{m}, \overline{\mathbf{m}}$ are complex conjugate of each other), if the tetrad vector $k^{\mu}$ is a principal null direction, then the algebraically special metrics automatically give $\Psi_{0}=0$. For the algebraically special metrics, the special cases are as follows:

Type II:

$$
\begin{aligned}
& \Psi_{0}=0=\Psi_{1}, \\
& \Psi_{2} \neq 0 .
\end{aligned}
$$

Type III:

$$
\begin{aligned}
& \Psi_{0}=\Psi_{1}=\Psi_{2}=0, \\
& \Psi_{3} \neq 0 .
\end{aligned}
$$

Type $\mathrm{N}$ :

$$
\begin{aligned}
& \Psi_{0}=\Psi_{1}=\Psi_{2}=\Psi_{3}=0, \\
& \Psi_{4} \neq 0 .
\end{aligned}
$$

Type D:

$$
\begin{aligned}
& \Psi_{0}=\Psi_{1}=\Psi_{3}=\Psi_{4}=0, \\
& \Psi_{2} \neq 0 .
\end{aligned}
$$

Penrose proposed a Cosmic Censorship Conjecture (CCC) [3-5] to forbid the occurrence of naked singularities in a solution of the field equations. According to the Weak Cosmic Censorship, singularities have no effect on distant observers; that is, they cannot communicate with far-away observers. Till date, there are no mathematical theorems or proofs that support (or counter) this conjecture. On the contrary, there is no mathematical reason that a naked singularity cannot exist in a solution of the field equations. Therefore, the formulation and proof (or disproof) of this conjecture remain to be one of the unsolved problems in General Relativity. Attempts have been made to provide the theoretical 
framework to devise a technique to distinguish between black holes and naked singularities from astrophysical data mainly through gravitational lensing (GL). Some significant works in this direction are the study of strong gravitational lensing in the Janis-Newman-Winicour space-time [6,7] and its rotating generalization [8] and notably the work in [9-12]. Other workers have shown that naked singularities and black holes are differentiated by the properties of the accretion disks that accumulate around them. Consequently, the study of naked singularities and space-time with such objects is of considerable current interest. In [13], the authors have enumerated three possible end states of gravitational collapse. There are a number of examples of gravitational collapse in spherically symmetric that formed naked singularities known. An earliest model that admits both naked and covered singularities is the Lemaitre-Tolman-Bondi (LTB) [14-16] solutions, a spherically symmetric inhomogeneous collapse of dust fluid. Papapetrou [17] pointed out the formation of naked singularities in Vaidya [18] radiating solution, a null dust fluid space-time generated from Schwarzschild vacuum solution. A small sample of spherically symmetric gravitational collapse solutions with a naked singularities are in [19-30]. Thus, the theoretical existence of naked singularities would mean that the gravitational collapse may be observable from the rest of the space-time.

In the present article, an axially symmetric solution of the field equations with a naked curvature singularity will be presented. The gravitational collapsing of cylindrically symmetric models that formed a naked singularity has been discussed in [31, 32]. Apostolatos and Thorne [33] investigated the collapse of counter-rotating dust shell cylinder and showed that rotation, even if it is infinitesimally small, can halt the gravitational collapse of the cylinder. Echeverria [34] studied the evolution of a cylindrical dust shell analytically at late times and numerically for all times. Guttia et al. [35] studied the collapse of nonrotating, infinite dust cylinders. Nakao and Morisawa [36] studied the high-speed collapse of cylindrically symmetric thick shell composed of dust and perfect fluid with nonvanishing pressure [37]. Recent work describes the cylindrically symmetric collapse of counterrotating dust shells [38-40], self-similar scalar field [41], axially symmetric vacuum space-time [42], and a cylindrically symmetric anisotropic fluid space-time [43]. Some other examples of nonspherical gravitational collapse would be discussed in [44-53].

The Einstein field equations (taking cosmological constant $\Lambda=0$ ) are given by

$$
R_{\mu \nu}-\frac{1}{2} g_{\mu \nu} R=T_{\mu \nu}, \quad \mu, \nu \in(0,1,2,3)
$$

where $R_{\mu \nu}$ is the Ricci tensor, $R$ is the Ricci scalar, and $T_{\mu \nu}$ is the energy-momentum tensor.

Pure radiation or null dust fields are the fields of massless radiation which is considered as the incoherent superposition of waves with random phases and different polarization but with the same propagation direction. The radiation can arise from fields of different types, from electromagnetic null fields, massless scalar fields, and neutrino fields, or from the high frequency limit of gravitational waves. The energymomentum tensor of pure radiation field [54] is

$$
T^{\mu \nu}=\rho k^{\mu} k^{\nu}=2 \Phi_{22} k^{\mu} k^{\nu}, \quad k_{\mu} k^{\mu}=0,
$$

where $\rho$ is the energy density of null dust (pure radiation field) and $k^{\mu}$ is the null vector.

\section{Analysis of the Null Dust Space-Time}

Consider the following axially symmetric metric in $(t, r, \phi, z)$ coordinates:

$$
\begin{aligned}
d s^{2}= & g_{r r} d r^{2}+g_{\phi \phi} d \phi^{2}+2 g_{t \phi} d t d \phi+2 g_{z \phi} d z d \phi \\
& +g_{z z} d z^{2}
\end{aligned}
$$

where the different metric functions are

$$
\begin{aligned}
& g_{r r}=\operatorname{sech}^{4} r \tanh r, \\
& g_{\phi \phi}=-\tanh ^{2} r \sinh t, \\
& g_{\phi t}=g_{t \phi}=g_{\phi \phi}^{\prime}, \\
& g_{\phi z}=g_{z \phi}=z \tanh ^{2} r, \\
& g_{z z}=\operatorname{coth} r,
\end{aligned}
$$

where prime denotes derivate with respect to time, $g_{\phi \phi}^{\prime}=$ $d g_{\phi \phi} / d t$. Here $\phi$ coordinate is assumed periodic $\phi \in[0,2 \pi)$. We have labelled the coordinates $x^{0}=t, x^{1}=r, x^{2}=\phi$, and $x^{3}=z$. The ranges of the other coordinates are $-\infty<$ $t<\infty, 0 \leq r<\infty$, and $-\infty<z<\infty$. The metric is Lorentzian with signature $(+,+,+,-)$ and the determinant of the corresponding metric tensor $g_{\mu \nu}$ is

$$
\operatorname{det} g=-\tanh ^{4} r \operatorname{sech}^{4} r \cosh ^{2} t .
$$

For space-time (7), the Ricci scalar $R=0$ and the nonzero component of the Ricci tensor $R^{\mu \nu}$ is

$$
R_{\phi \phi}=\frac{1}{2} \tanh ^{3} r
$$

For space-time (7), the null vector is defined by $k_{\mu}=$ $(0,0,1,0)$. Therefore, the nonzero component of the energymomentum tensor (6) is

$$
T_{\phi \phi}=\rho .
$$

From the field equation (5) using (10) and (11), we get

$$
\rho(r)=\frac{1}{2} \tanh ^{3} r
$$

The energy density of null dust satisfies the null energy condition (NEC).

The curvature scalar invariants for metric (7) are

$$
\begin{aligned}
R^{\mu \nu \rho \sigma} R_{\mu \nu \rho \sigma} & =12 \operatorname{coth}^{6} r, \\
R^{\mu \nu \rho \sigma ; \tau} R_{\mu \nu \rho \sigma ; \tau} & =180 \operatorname{coth}^{9} r .
\end{aligned}
$$


From the above equation, it is clear that the scalar curvature invariants and first-order differential invariants diverge (blow up) on the symmetry axis $r=0$, indicating the existence of a naked curvature singularity. Since the naked curvature singularity occurs without an event horizon, the Cosmic Censorship Conjecture has no physical interest.

2.1. Geodesics in the Neighborhood of the Singularity. To discuss the geodesics motion of free test particles which necessarily hit the singularity $r=0$, one needs to derive expression for their paths. Here we focus on radial geodesics on the symmetry axis $r=0$ [55].

The Lagrangian for the metric (7) is given by

$$
\begin{aligned}
£ & =\frac{1}{2} g_{\mu \nu} \dot{x}^{\mu} \dot{x}^{\nu} \\
& =\frac{1}{2}\left[g_{r r} \dot{r}^{2}+g_{z z} \dot{z}^{2}+g_{\phi \phi} \dot{\phi}^{2}+2 g_{t \phi} \dot{t} \dot{\phi}+2 g_{z \phi} \dot{z} \dot{\phi}\right],
\end{aligned}
$$

where dot stands for derivative with respect to an affine parameter. From (7) and (14), it is clear that $\phi$ is a cyclic coordinate. There exists constant of motion corresponding to this cyclic coordinate; that is, the azimuthal angular momentum $p_{\phi}$ is a constant given by

$$
p_{\phi}=\text { const }=g_{z \phi} \dot{z}+g_{\phi \phi} \dot{\phi}+g_{t \phi} \dot{t} .
$$

For metric (7), geodesics equations for $t$ and $r$ coordinates are

$$
\begin{aligned}
\ddot{t}= & \operatorname{sech} t\left\{-\frac{1}{2} z^{2} \tanh ^{3} r \dot{\phi}^{2}+\dot{z}(3 z \operatorname{csch} r \operatorname{sech} r \dot{r}+\dot{z})\right\} \\
& -(2 \operatorname{csch} r \operatorname{sech} r \dot{r}+\dot{\phi}) \dot{t}-\frac{1}{2} \tanh t\left(\dot{\phi}^{2}+2 \dot{t}^{2}\right), \\
\ddot{r}= & \frac{1}{2}\left[-\operatorname{sech} r \operatorname{csch} r \dot{r}^{2}+4 \tanh r \dot{r}^{2}-\cosh ^{2} r\right. \\
& \left.\cdot\left\{2 \sinh t \dot{\phi}^{2}-4 z \dot{\phi} \dot{z}+\operatorname{coth}^{3} r \dot{z}^{2}+4 \cosh t \dot{\phi} \dot{t}\right\}\right] .
\end{aligned}
$$

For radial geodesics we have $\dot{z}=0=\dot{\phi}$. From (16), we get

$$
\begin{aligned}
& \ddot{t}=-2 \operatorname{csch} r \operatorname{sech} r \dot{r} \dot{t}-\tanh t \dot{t}^{2}, \\
& \ddot{r}=\frac{1}{2}(-\operatorname{csch} r \operatorname{sech} r+4 \tanh r) \dot{r}^{2} .
\end{aligned}
$$

The solution of (17) is as follows:

$$
\begin{aligned}
& \dot{t}(s)=c_{1} \operatorname{sech} t \operatorname{coth}^{2} r, \quad c_{1}>0, \\
& \dot{r}(s)=c_{2} \cosh ^{2} r \operatorname{coth}^{1 / 2} r, \quad c_{2}>0 .
\end{aligned}
$$

Again solving (18) yields

$$
\begin{aligned}
& t(s)=\sinh ^{-1}\left[c_{4}-\frac{3 c_{1}}{c_{2}}\left(\frac{2}{3}\right)^{4 / 3}\left(c_{3}+c_{2} s\right)^{-1 / 3}\right], \\
& r(s)=\tanh ^{-1}\left[\left(\frac{3}{2}\right)^{2 / 3}\left(c_{3}+c_{2} s\right)^{2 / 3}\right],
\end{aligned}
$$

where $c_{i}, i=1, \ldots, 4$, are arbitrary constants. Thus from (19), it is clear that the geodesic path $t$ is complete for finite value of the affine parameter $s$ except at $s=0$ (taking $c_{3}=0$ ), where it is unbounded. Therefore, the presented space-time is radially geodesically incomplete.

2.2. Strength of Naked Singularities. The strength of singularity, which is the measure of its destructive capacity, is the most important feature. To determine the strength of naked singularities as strong and weak types, we consider the criteria developed by Tipler [56] and Krolak [57], which provide insights into the magnitude of tidal forces experienced by an in-falling detector (or an observer) towards the singularity [58]. It is widely believed that the analytical extension of the space-time through a singularity is not possible, if it satisfies the strong curvature condition.

A naked singularity (NS) is said to be strong if the collapsing objects do get crushed to a zero volume at the singularity and a weak one if they do not $[21,59]$. Following Clarke and Krolak [58], a sufficient condition for a singularity to be strong in the sense of Tipler [56] is that at least along one radial geodesic we must have

$$
\lim _{s \rightarrow 0} s^{2} R_{\mu \nu} \frac{d x^{\mu}}{d s} \frac{d x^{\nu}}{d s} \neq 0(>0),
$$

where $d x^{\mu} / d s$ is the tangent vector to the radial geodesics and $R_{\mu \nu}$ is the Ricci tensor. The weaker condition, which we called the limiting focusing condition [57], is defined by

$$
\lim _{s \rightarrow 0} s R_{\mu \nu} \frac{d x^{\mu}}{d s} \frac{d x^{\nu}}{d s} \neq 0 .
$$

Hence from (20), we have the strong curvature condition for space-time (7)

$$
\begin{aligned}
\lim _{s \rightarrow 0} s^{2}\left[R_{r r}\left(\frac{d r}{d s}\right)^{2}+R_{t t}\left(\frac{d t}{d s}\right)^{2}\right] \\
=\lim _{s \rightarrow 0} s^{2}\left[0 \times\left(\frac{d r}{d s}\right)^{2}+0 \times\left(\frac{d t}{d s}\right)^{2}\right]=0 .
\end{aligned}
$$

Similarly, one can calculate the limiting focusing condition, and it also becomes zero. Thus the naked singularity (NS) which is formed due to the curvature singularity satisfies neither the strong curvature condition nor the limiting focusing condition. Therefore, the analytical extension of the space-time through the singularity is possible.

2.3. Equation of Orbit and Gravitational Lensing. We calculate first the equation of orbit for $r(\phi)$. Here we restrict our discussion to the orbital motion of the free test particle which moves in the $z=$ const-plane. To get an equation for $r(\phi)$ we start with

$$
r^{\prime}(\phi)=\frac{d r}{d \phi}=\frac{\dot{r}}{\dot{\phi}} .
$$

To work out the lens equation we have to calculate the null geodesics in $z=$ const-planes. From the null geodesics $d s^{2}=$ 0 using space-time (7) we have 


$$
g_{r r} \dot{r}^{2}+g_{\phi \phi} \dot{\phi}^{2}+2 g_{t \phi} \dot{t} \dot{\phi}=0
$$

where we have chosen $z=z_{0}$ (where $z_{0}$ is a constant equal to zero).

We define angular velocity $\Omega$ of a zero angular momentum particle as measured by an observer (ZAMO) in $z=$ const-planes. The angular velocity $\Omega$ (the angular velocity of the frame dragging) is defined by $[60,61]$

$$
\Omega=\frac{\dot{\phi}}{\dot{t}}=-\frac{g_{t \phi}}{g_{\phi \phi}}=-\frac{g_{\phi \phi}^{\prime}}{g_{\phi \phi}}=-\operatorname{coth} t, \quad t<0 .
$$

Therefore, from (24) using (25), one can obtain the following equation for the photon orbit:

$$
\begin{aligned}
g_{r r} \dot{r}^{2}-g_{\phi \phi} \dot{\phi}^{2} & =0 \Longrightarrow \\
\left(\frac{\dot{r}}{\dot{\phi}}\right)^{2} & =\frac{g_{\phi \phi}}{g_{r r}} \Longrightarrow \\
\frac{\dot{r}}{\dot{\phi}} & = \pm \sqrt{\frac{g_{\phi \phi}}{g_{r r}}} \Longrightarrow \\
\frac{d r}{d \phi} & = \pm \sqrt{\frac{g_{\phi \phi}}{g_{r r}}}= \pm \sqrt{H(r, t)}
\end{aligned}
$$

Substituting $g_{\phi \phi}$ and $g_{r r}$ and integrating (26) immediately give the azimuthal angle $\phi$ as a function of $r$

$$
\phi= \pm 2 \sqrt{-\operatorname{csch} t} \tanh ^{1 / 2} r= \pm 2 \operatorname{csch}^{1 / 2} T \tanh ^{1 / 2} r
$$

where $t=-T<0, T>0$. Here we have set $\phi(r=\infty)=0$. Therefore, the Einstein deflection angle $\widehat{\boldsymbol{\alpha}}$ is

$$
\begin{aligned}
\widehat{\boldsymbol{\alpha}}\left(r_{0}\right) & =2 \int_{r=r_{0}}^{\infty} \frac{d r}{\sqrt{H(r, t)}}-\pi \\
& =4 \operatorname{csch}^{1 / 2} T\left(1-\tanh ^{1 / 2} r_{0}\right)-\pi .
\end{aligned}
$$

2.4. Closed Timelike Curves of the Space-Time: A Cosmic Time Machine. The presented space-time admits closed timelike curves which appear after a certain instant of time. There are many solutions of the field equations admitting closed timelike curves known (see references in [62]).

Consider an azimuthal curve $\gamma$ defined by $r=r_{0}, z=z_{0}$, and $t=t_{0}$, where $r_{0}>0, z_{0}$, and $t_{0}$ are constants. From the line element (7) we have

$$
d s^{2}=-\sinh t \tanh ^{2} r d \phi^{2}
$$

These curves are null at $t=t_{0}=0$ and spacelike throughout $t=t_{0}<0$ but become timelike for $t=t_{0}>0$, which indicates the presence of closed timelike curves (CTCs). Hence CTCs form at a definite instant of time satisfying $t=t_{0}>0$. The above analysis is valid provided that the CTCs evolve from an initial spacelike $t=$ const hypersurface (thus $t$ is a time coordinate) [63]. This can be determined by calculating the norm of the vector $\nabla_{\mu} t$ (or by determining the sign of $g^{t t}$ in the metric tensor $g^{\mu \nu}$ [63]). A hypersurface $t=$ const is spacelike provided $g^{t t}<0$ for $t<0$, timelike provided $g^{t t}>0$ for $t>0$, and null hypersurface provided $g^{t t}=0$ for $t=0$. In our case, from metric (7) we found that

$$
g^{t t}=\nabla_{\mu} t \nabla^{\mu} t=\operatorname{sech}^{2} t \operatorname{coth}^{2} r\left(z^{2} \tanh ^{3} r+\sinh t\right) .
$$

Here we have chosen constant $z$-planes defined by $z=z_{0}$, where $z_{0}$ is a constant equal to zero. Thus, the hypersurface $t=$ const $=t_{0}<0$ is spacelike $(r \neq 0)$ and can be chosen as initial conditions over which the initial data may be specified. There is a Cauchy horizon at $t=t_{0}=0$ for any such initial spacelike hypersurface $t=t_{0}<0$. The null curve $t=t_{0}=0$ serves as the Chronology horizon which divided the space-time into a nonchronal region with CTCs and a chronal region without CTCs. The Chronology horizon is a special type of Cauchy horizon which has been discussed in detail in $[64,65]$. Hence the space-time evolves from an initial spacelike hypersurface in a causally well-behave manner up to a moment, that is, a null hypersurface $t=t_{0}=0$, and the formation of CTCs takes place from a causally well-behaved initial condition.

The possibility that a naked curvature singularity gives rise to a Cosmic Time Machine has been discussed by Clarke and de Felice [66] (see also [67-69]). A Cosmic Time Machine is a space-time which is asymptotically flat and admits closed nonspacelike curves which extend to future infinity. The author and collaborators constructed such Cosmic Time Machines [42, 43], quite recently. The presented time-dependent space-time may represent such a Cosmic Time Machine.

\section{Classification of the Space-Time and Effects on the Test Particles}

For classification of metric (7), one can construct a set of null tetrad vectors $(\mathbf{k}, \mathbf{l}, \mathbf{m}, \overline{\mathbf{m}})$. The metric tensor can be expressed as

$$
g_{\mu \nu}=-k_{\mu} l_{\nu}-l_{\mu} k_{\nu}+m_{\mu} \bar{m}_{\nu}+\bar{m}_{\mu} m_{\nu}
$$

where the tetrad vectors are orthogonal except for $k_{\mu} l^{\mu}=-1$ and $m_{\mu} \bar{m}^{\mu}=1$. The nonzero Weyl scalars using the set of null tetrad are

$$
\begin{aligned}
& \Psi_{2}=C_{\mu \nu \rho \sigma} k^{\mu} m^{\nu} \bar{m}^{\rho} l^{\sigma}=\frac{1}{2 \tanh ^{3} r}, \\
& \Psi_{4}=C_{\mu \nu \rho \sigma} l^{\mu} \bar{m}^{\nu} l^{\rho} \bar{m}^{\sigma}=-\frac{1}{4} \tanh ^{3} r-i \frac{3 z}{4} \tanh r,
\end{aligned}
$$

while others are $\Psi_{0}=0=\Psi_{1}=\Psi_{3}$.

We set up an orthonormal frame $\mathbf{e}_{(a)}=\left\{\mathbf{e}_{(0)}, \mathbf{e}_{(1)}, \mathbf{e}_{(2)}\right.$, $\left.\mathbf{e}_{(3)}\right\}, \mathbf{e}_{(a)} \cdot \mathbf{e}_{(b)} \equiv e_{(a)}{ }^{\mu} e_{(b)}{ }^{\nu} g_{\mu \nu}=\eta_{(a)(b)}=\operatorname{diag}(-1,+1,+1,+1)$, which consists of one timelike vector $\mathbf{e}_{(0)}$ and three spacelike unit vectors $\mathbf{e}_{(i)}, i=1,2,3$. Notation is such that small Latin indices are raised and lowered with $\eta^{(a)(b)}, \eta_{(a)(b)}$, and Greek indices are raised and lowered with $g^{\mu \nu}$ and $g_{\mu \nu}$. These 
frame components can conveniently be expressed using the corresponding null tetrad vectors as

$$
\begin{aligned}
& \mathbf{e}_{(0)}=\frac{1}{\sqrt{2}}(\mathbf{k}+\mathbf{l}), \\
& \mathbf{e}_{(1)}=\frac{1}{\sqrt{2}}(\mathbf{m}+\overline{\mathbf{m}}), \\
& \mathbf{e}_{(2)}=\frac{1}{\sqrt{2}}(\mathbf{k}-\mathbf{l}), \\
& \mathbf{e}_{(3)}=\frac{-i}{\sqrt{2}}(\mathbf{m}-\overline{\mathbf{m}}) .
\end{aligned}
$$

In order to analyze the effects of the gravitational field of the above vacuum solution, it is natural to investigate the specific influence of various components of these fields on the relative motion of the free test particles. Such a local characterization of space-time, based on the equation of geodesic deviation frame, was described by Pirani $[70,71]$ and Szekeres [72, 73] (see also [74, 75])

$$
\frac{D^{2} Z^{\mu}}{d \tau^{2}}=-R_{\nu \rho \sigma}^{\mu} U^{\nu} Z^{\rho} U^{\sigma}
$$

where $\mathbf{U} \cdot \mathbf{U}=-1$ is the timelike four-velocity of the free test particle (observer) and $Z(\tau)$ is the displacement vector, where $\tau$ is the proper time. By projecting (34) onto the orthonormal frame $\mathbf{e}_{(a)}$ given by (33), we get

$$
\ddot{Z}^{(i)}=-R_{(0)(j)(0)}^{(i)} Z^{(j)},
$$

where $i, j=1,2,3, \mathbf{e}_{(0)}=U$, and

$$
R_{(0)(j)(0)}^{(i)}=e_{(i)}^{\mu} U^{\nu} e_{(j)}^{\rho} U^{\sigma} R_{\mu \nu \rho \sigma}
$$

The frame components of the displacement vector $Z^{(j)} \equiv$ $e_{\mu}^{(i)} Z^{\mu}$ determine directly the distance between close test particles. Their physical relative acceleration is given by

$$
\ddot{Z}^{(i)}=e_{\mu}^{(i)}\left(\frac{D^{2} Z^{\mu}}{d \tau^{2}}\right) .
$$

Equation (34) also implies

$$
\ddot{Z}^{(0)}=-U_{\mu}\left(\frac{D^{2} Z^{\mu}}{d \tau^{2}}\right)=R_{\mu \nu \rho \sigma} U^{\mu} U^{\nu} Z^{\rho} U^{\sigma}=0
$$

so that $Z^{(0)}=a_{0} \tau+b_{0}, a_{0}, b_{0}$ are constants. Setting $Z^{(0)}=0$, all test particles are synchronized by the proper time. From the standard definition of the Weyl tensor using the metric (7), we get

$$
R_{(i)(0)(j)(0)}=C_{(i)(0)(j)(0)}+\frac{1}{2}\left\{\delta_{i j} T_{(0)(0)}-T_{(i)(j)}\right\} .
$$

For space-time (7), the only nonvanishing scalars are given by (32) so that

$$
\begin{aligned}
C_{(1)(0)(1)(0)} & =\frac{1}{2} \mathfrak{R} e \Psi_{4}-\mathfrak{R} e \Psi_{2}, \\
C_{(2)(0)(2)(0)} & =-\frac{1}{2} \mathfrak{R} e \Psi_{4}-\mathfrak{R} e \Psi_{2}, \\
C_{(1)(0)(2)(0)} & =-\frac{1}{2} \mathfrak{I} m \Psi_{4}, \\
C_{(3)(0)(3)(0)} & =2 \mathfrak{R} e \Psi_{2}, \\
T_{(0)(0)} & =T_{(2)(2)} \equiv \boldsymbol{\Phi}_{\mathbf{2 2}} .
\end{aligned}
$$

Therefore, the equation of geodesic deviation (20) takes the form

$$
\begin{aligned}
\ddot{Z}^{(1)}= & -R_{(0)(j)(0)}^{(1)} Z^{(j)} \\
= & -\left[C_{(1)(0)(1)(0)}+\frac{1}{2} T_{(0)(0)}\right] Z^{(1)}-C_{(1)(0)(2)(0)} Z^{(2)} \\
= & -\frac{1}{2} \mathfrak{R} e \Psi_{4} Z^{(1)}+\frac{1}{2} \Im m \Psi_{4} Z^{(2)}+\mathfrak{R} e \Psi_{2} Z^{(1)} \\
& -\frac{1}{2} \Phi_{22} Z^{(1)} \\
= & -\mathbf{A}_{+} Z^{(1)}+\mathbf{A}_{\times} Z^{(2)}+\mathbf{C} Z^{(1)}-\frac{1}{2} \Phi_{22} Z^{(1)}, \\
\ddot{Z}^{(2)}= & -R_{(0)(j)(0)}^{(2)} Z^{(j)}=-C_{(2)(0)(2)(0)} Z^{(2)} \\
= & \frac{1}{2} \Re_{e} \Psi_{4} Z^{(2)}+\mathfrak{R}_{e} \Psi_{2} Z^{(2)}=\mathbf{A}_{+} Z^{(2)}+\mathbf{C} Z^{(2)}, \\
\ddot{Z}^{(3)}= & -R_{(0)(j)(0)}^{(3)} Z^{(j)}=-\left[C_{(3)(0)(3)(0)}+\frac{1}{2} T_{(0)(0)}\right] Z^{(3)} \\
= & -2 \mathbf{C} Z^{(3)}-\frac{1}{2} \Phi_{22} Z^{(3)},
\end{aligned}
$$

where $\mathbf{A}_{+}=(1 / 2) \mathfrak{R} e \Psi_{4}, \mathbf{A}_{\times}=(1 / 2) \mathfrak{I} m \Psi_{4}, \mathbf{C}=\mathfrak{R} e \Psi_{2}$, and $\mathfrak{R} e$ and $\Im m$ stand for real and complex, respectively. The above equations are well suited for physical interpretation. Clearly, the relative motions of nearby test particles depend on the following:

(1) The local free gravitational field, consisting of two components. There are a Coulomb-component with amplitude $\mathbf{C}$ and transverse wave components with amplitudes $\mathbf{A}_{+}$ and $\mathbf{A}_{\times}$of two polarization modes “+" and " $\times$" representing the effect of gravitational waves on the particles in the presented type II space-time.

(2) The terms describing the matter-content pure radiation field $\left(\Phi_{22}\right)$ which affects the motion only in the transverse plane spanned by the vectors $\mathbf{e}_{(1)}$ and $\mathbf{e}_{(3)}$.

\section{Conclusions}

We presented an axially symmetric time-dependent solution of the field equations which possesses a naked curvature singularity on the symmetry axis. The naked singularity 
occurs without an event horizon; therefore the Cosmic Censorship has no physical interest. The space-time satisfies null dust as that for matter-energy content with positive energy density which is finite on the symmetry axis and obeys the null energy condition. The radial geodesics of the space-time near the singularity were discussed and it was found that these are incomplete. The analytical extension of the space-time through the singularity is possible since the strength of the naked curvature singularity fails to satisfy the strong curvature condition. We obtained the equation for the photon orbit and subsequently the deflection angle $\widehat{\boldsymbol{\alpha}}$ which is found time-dependent. The presented space-time admits closed timelike curves which develop at some particular moment from an initial spacelike hypersurface in a causally well-behaved manner. The possibility that a naked curvature singularity gives rise to a Cosmic Time Machine has been discussed by Clarke and de Felice [66]. The presented spacetime may represent such a Cosmic Time Machine. Finally, the physical interpretation of the presented solution, based on the study of the equation of the geodesic deviation, was presented. It was demonstrated that the solution represents exact gravitational waves consisting of two components, transverse wave components with amplitudes $\mathbf{A}_{+}$and $\mathbf{A}_{\times}$ of two polarization modes " + " and " $x$ " and Coulomb-like components of amplitude $\mathbf{C}$. There is a matter-energy null dust field interaction with the test particles.

\section{Conflicts of Interest}

The author declares that there are no conflicts of interest.

\section{References}

[1] E. Newman and R. Penrose, "An approach to gravitational radiation by a method of spin coefficients," Journal of Mathematical Physics, vol. 3, pp. 566-578, 1962.

[2] E. Newman and R. Penrose, "Errata: an approach to gravitational radiation by a method of spin coefficients," Journal of Mathematical Physics, vol. 4, 998 pages, 1963.

[3] R. Penrose, "Gravitational collapse: the role of general relativity," Rivista del Nuovo Cimento, vol. 1, pp. 252-276, 1969.

[4] R. Penrose, "Singularities and time-asymetry," in General Relativity: An Einstein Centenary Survey, S. W. Hawking and W. Israel, Eds., pp. 581-638, Cambridge University Press, Cambridge, 1979.

[5] R. Penrose, "The question of cosmic censorship," in Black Holes and Relativistic Stars, R. M. Wald, Ed., Chicago University Press, Chicago, Ill, USA, 1994.

[6] K. S. Virbhadra, D. Narasimha, and S. M. Chitre, "Role of scalar field in gravitational lensing," Astronomy and Astrophysics, vol. 337, pp. 1-8, 1998.

[7] K. S. Virbhadra and G. F. R. Ellis, "Gravitational lensing by naked singularities," Physical Review D, vol. 65, no. 10, Article ID 103004, 2002.

[8] G. N. Gyulchev and S. S. Yazadjiev, Physical Review D, vol. 78, Article ID 083004, 2008.

[9] M. Werner and A. Petters, "Magnification relations for Kerr lensing and testing cosmic censorship," Physical Review D, vol. 76, Article ID 064024, 2007.
[10] C. Bambi and N. Yoshida, "Shape and position of the shadow in the $\delta=2$ Tomimatsu-Sato spacetime," Classical and Quantum Gravity, vol. 27, no. 20, Article ID 205006, 2010.

[11] C. Bambi and K. Freese, "Apparent shape of super-spinning black holes," Physical Review D. Particles, Fields, Gravitation, and Cosmology, vol. 79, no. 4, Article ID 043002, 2009.

[12] K. Hioki and K.-i. Maeda, "Bulk spacetimes for cosmological braneworlds with a time-dependent extra dimension," Physical Review D, vol. 80, Article ID 024042, 2009.

[13] A. N. Chowdhury, M. Patil, D. Malafarina, and P. Joshi, "Circular geodesics and accretion disks in the Janis-NewmanWinicour and gamma metric spacetimes," Physical Review D, vol. 85, Article ID 104031, 2012.

[14] G. Lemaitre, "L'univers en expansion," Annales de la Societe Scientifique de Bruxelles A, vol. 53, p. 51, 1933.

[15] R. C. Tolman, "Effect of inhomogeneity on cosmological models," Proceedings of the National Academy of Sciences of the United States of America, vol. 20, p. 169, 1934.

[16] H. Bondi, "Spherically symmetrical models in general relativity," Monthly Notices of the Royal Astronomical Society, vol. 107, p. $343,1947$.

[17] A. Papapetrou, A Random Walk in Relativity and Cosmology, Wiley, New York, NY, USA, 1985.

[18] P. C. Vaidya, “'Newtonian' Time in General Relativity," Nature, vol. 171, pp. 260-261, 1953.

[19] D. Christodoulou, "Violation of cosmic censorship in the gravitational collapse of a dust cloud," Communications in Mathematical Physics, vol. 93, pp. 171-195, 1984.

[20] P. S. Joshi, Global Aspects in Gravitation and Cosmology, vol. 87 of International Series of Monographs on Physics, The Clarendon Press, Oxford, UK, 1993.

[21] P. S. Joshi, Singularities, Black Holes and Cosmic Censorship, IUCAA publication, Pune, India, 1997.

[22] S. S. Deshingkar, I. H. Dwivedi, and P. S. Joshi, "Physical nature of the central singularity in spherical collapse," Physical Review $D$, vol. 59, Article ID 044018, 1999.

[23] S. Barve, T. P. Singh, C. Vaz, and L. Witten, "A simple derivation of the naked singularity in spherical dust collapse," Classical and Quantum Gravity, vol. 16, no. 6, pp. 1727-1732, 1999.

[24] P. S. Joshi and I. H. Dwivedi, "Naked singularities in spherically symmetric inhomogeneous Tolman-Bondi dust cloud collapse," Physical Review D, vol. 47, no. 12, pp. 5357-5369, 1993.

[25] P. S. Joshi and I. H. Dwivedi, "Initial data and the end state of spherically symmetric gravitational collapse," Classical and Quantum Gravity, vol. 16, no. 1, pp. 41-59, 1999.

[26] K. S. Govinder and M. Govender, "Gravitational collapse of null radiation and a string fluid," Physical Review D, vol. 68, no. 2, Article ID 024034, 2003.

[27] E. N. Glass and J. P. Krisch, "Radiation and string atmosphere for relativistic stars," Physical Review D, vol. 57, p. 5945, 1998.

[28] J. F. Villas da Rocha, "Type II fluid solutions to Einstein's field equations in $N$-dimensional spherical spacetimes," International Journal of Modern Physics D, vol. 11, no. 1, pp. 113-124, 2002.

[29] L. Herrera, A. Di Prisco, and J. Ospino, “The space-time outside a source of gravitational radiation: the axially symmetric null fluid," The European Physical Journal C, vol. 76, p. 603, 2016.

[30] A. Krasinski, Inhomogeneous Cosmological Models, Cambridge University Press, Cambridge, 1997.

[31] K. S. Thorne, Magic without Magic: John Archibald Wheeler, Freeman and Co., San Francisco, CA, USA, 1972. 
[32] S. A. Hayward, "Gravitational waves, black holes and cosmic strings in cylindrical symmetry," Classical and Quantum Gravity, vol. 17, p. 1749, 2000.

[33] T. A. Apostolatos and K. S. Thorne, "Rotation halts cylindrical, relativistic gravitational collapse," Physical Review. D. Third Series, vol. 46, no. 6, pp. 2435-2444, 1992.

[34] F. Echeverria, "Gravitational collapse of an infinite, cylindrical dust shell," Physical Review D, vol. 47, no. 6, pp. 2271-2282, 1993.

[35] S. Guttia, T. P. Singh, P. A. Sundararaj, and C. Vaz, "Gravitational collapse of an infinite dust cylinder," https://arxiv.org/abs/grqc/0212089.

[36] K.-I. Nakao and Y. Morisawa, "High speed dynamics of collapsing cylindrical dust fluid," Classical and Quantum Gravity, vol. 21, no. 8, pp. 2101-2113, 2004.

[37] K. Nakao and Y. Morisawa, "High-speed cylindrical collapse of perfect fluid," Progress of Theoretical Physics, vol. 113, p. 73, 2005.

[38] S. M. Goncalves and S. Jhingan, "A note on the cylindrical collapse of counter-rotating dust," International Journal of Modern Physics. D. Gravitation, Astrophysics, Cosmology, vol. 11, no. 9, pp. 1469-1477, 2002.

[39] B. C. Nolan, "Naked singularities in cylindrical collapse of counterrotating dust shells," Physical Review D, vol. 65, no. 10, Article ID 104006, 2002.

[40] P. R. C. T. Periera and A. Wang, "Gravitational collapse of cylindrical shells made of counterrotating dust particles," Physical Review D, vol. 62, Article ID 124001, 2000.

[41] A. Wang, "Critical collapse of a cylindrically symmetric scalar field in four-dimensional Einstein's theory of gravity," Physical Review D, vol. 68, no. 6, Article ID 064006, 12 pages, 2003.

[42] D. Sarma, F. Ahmed, and M. Patgiri, "Axially symmetric, asymptotically flat vacuum metric with a naked singularity and closed timelike curves," Advances in High Energy Physics, vol. 2016, Article ID 2546186, 4 pages, 2016.

[43] F. Ahmed, "Cylindrically symmetric, asymptotically flat, petrov type $\mathrm{d}$ spacetime with a naked curvature singularity and matter collapse," Advances in High Energy Physics, vol. 2017, Article ID 7943649, 6 pages, 2017.

[44] T. Chiba, "Cylindrically symmetric, asymptotically flat, petrov type D spacetime with a naked curvature singularity and matter collapse," Progress of Theoretical Physics, vol. 95, p. 321, 1996.

[45] T. Piran, "Cylindrical general relativistic collapse," Physical Review Letters, vol. 41, p. 1085, 1978.

[46] K. S. Thorne, "Energy of infinitely long, cylindrically symmetric systems in general relativity," Physical Review B, vol. 138, p. B251, 1965.

[47] T. A. Morgan, General Relativity and Gravitation, vol. 4, no. 4, pp. 273-278, 1973.

[48] P. S. Letelier and A. Z. Wang, "Singularities formed by the focusing of cylindrical null fluids," Physical Review. D. Third Series, vol. 49, no. 10, pp. 5105-5110, 1994.

[49] J. M. Senovilla and R. Vera, "Cylindrically symmetric dust spacetime," Classical and Quantum Gravity, vol. 17, no. 14, pp. 2843-2846, 2000.

[50] H. Bondi, "The mass of cylindrical systems in general relativity," Proceedings of the Royal Society. London. Series A. Mathematical, Physical and Engineering Sciences, vol. 427, no. 1873, pp. 259264, 1990.

[51] M. A. Melvin, "Pure magnetic and electric geons," Physics Letters. B, vol. 8, pp. 65-68, 1964.

[52] M. A. Melvin, "Dynamics of Cylindrical Electromagnetic Universes," Physical Review, vol. 139, p. B225, 1965.
[53] J. C. de Araujo and A. Wang, "Rigidly rotating dust in general relativity," General Relativity and Gravitation, vol. 32, no. 10, pp. 1971-1980, 2000.

[54] H. Stephani, D. Kramer, M. MacCallum, C. Hoenselaers, and E. Herlt, Exact Solutions of Einstein's Equations, Cambridge University Press, Cambridge, UK, 2003.

[55] Y. Kurita and K.-I. Nakao, "Naked singularity resolution in cylindrical collapse," Physical Review. D. Third Series, vol. 73, no. 6, Article ID 064022, 2006.

[56] F. J. Tipler, "Singularities in conformally flat spacetimes," Physics Letters A, vol. 64, no. 1, pp. 8-10, 1977.

[57] A. Krolak, "Towards the proof of the cosmic censorship hypothesis in cosmological space-times," Journal of Mathematical Physics, vol. 28, no. 1, pp. 138-141, 1987.

[58] C. J. Clarke and A. Krolak, "Conditions for the occurrence of strong curvature singularities," Journal of Geometry and Physics, vol. 2, no. 2, pp. 127-143, 1985.

[59] I. H. Dwivedi and P. S. Joshi, "On the nature of naked singularities in Vaidya spacetimes," Classical and Quantum Gravity, vol. 6, no. 11, pp. 1599-1606, 1989.

[60] P. Collas and D. Klein, "Frame dragging anomalies for rotating bodies," General Relativity and Gravitation, vol. 36, no. 5, pp. 1197-1206, 2004

[61] H. T. Mei and W. Y. Jiu, "Frame dragging in the field of Kerr family," Chinese Physics, vol. 15, no. 1, pp. 232-234, 2006.

[62] F. Ahmed, "Cylindrically symmetric pure radiation space-time and closed timelike geodesics," Progress of Theoretical and Experimental Physics, vol. 2017, Article ID 043E02, 2017.

[63] A. Ori, "A class of time-machine solutions with a compact vacuum core," Physical Review Letters, vol. 95, no. 2, Article ID 021101, 2005.

[64] S. W. Hawking, "Chronology protection conjecture," Physical Review D, vol. 46, no. 2, pp. 603-611, 1992.

[65] S. W. Hawking and G. F. R. Ellis, The Large Scale Structure of Space-Time, Cambridge University Press, London, UK, 1973.

[66] C. J. Clarke and F. de Felice, "Globally noncausal space-times. II. Naked singularities and curvature conditions," General Relativity and Gravitation, vol. 16, no. 2, pp. 139-148, 1984.

[67] F. de Felice, Lecture Notes in Physics, vol. 455, Springer-Verlag Publishers, 1995.

[68] F. de Felice, "Naked singularities, cosmic time machines and impulsive events," Nuovo Cimento B, vol. 122, p. 481, 2007.

[69] F. de Felice, "Cosmic time machines: the causality issue," EPJ Web of Conferences, vol. 58, Article ID 01001, 4 pages, 2013.

[70] F. A. E. Pirani, "On the physical significance of the Riemann tensor," Acta Physica Polonica. B, vol. 15, pp. 389-405, 1956.

[71] F. A. E. Pirani, "Invariant formulation of gravitational radiation theory," Physical Review, vol. 105, no. 3, pp. 1089-1099, 1957.

[72] P. Szekeres, “The gravitational compass," Journal of Mathematical Physics, vol. 6, pp. 1387-1391, 1965.

[73] P. Szekeres, "On the propagation of gravitational fields in matter," Journal of Mathematical Physics, vol. 7, p. 751, 1966.

[74] J. Bicák and J. Podolský, "Gravitational waves in vacuum spacetimes with cosmological constant. II. Deviation of geodesics and interpretation of nontwisting type $N$ solutions," Journal of Mathematical Physics, vol. 40, no. 9, pp. 4506-4517, 1999.

[75] J. Podolský and M. Ortaggio, "Explicit Kundt type II and $N$ solutions as gravitational waves in various type $D$ and $O$ universes," Classical and Quantum Gravity, vol. 20, no. 9, pp. 1685-1701, 2003. 

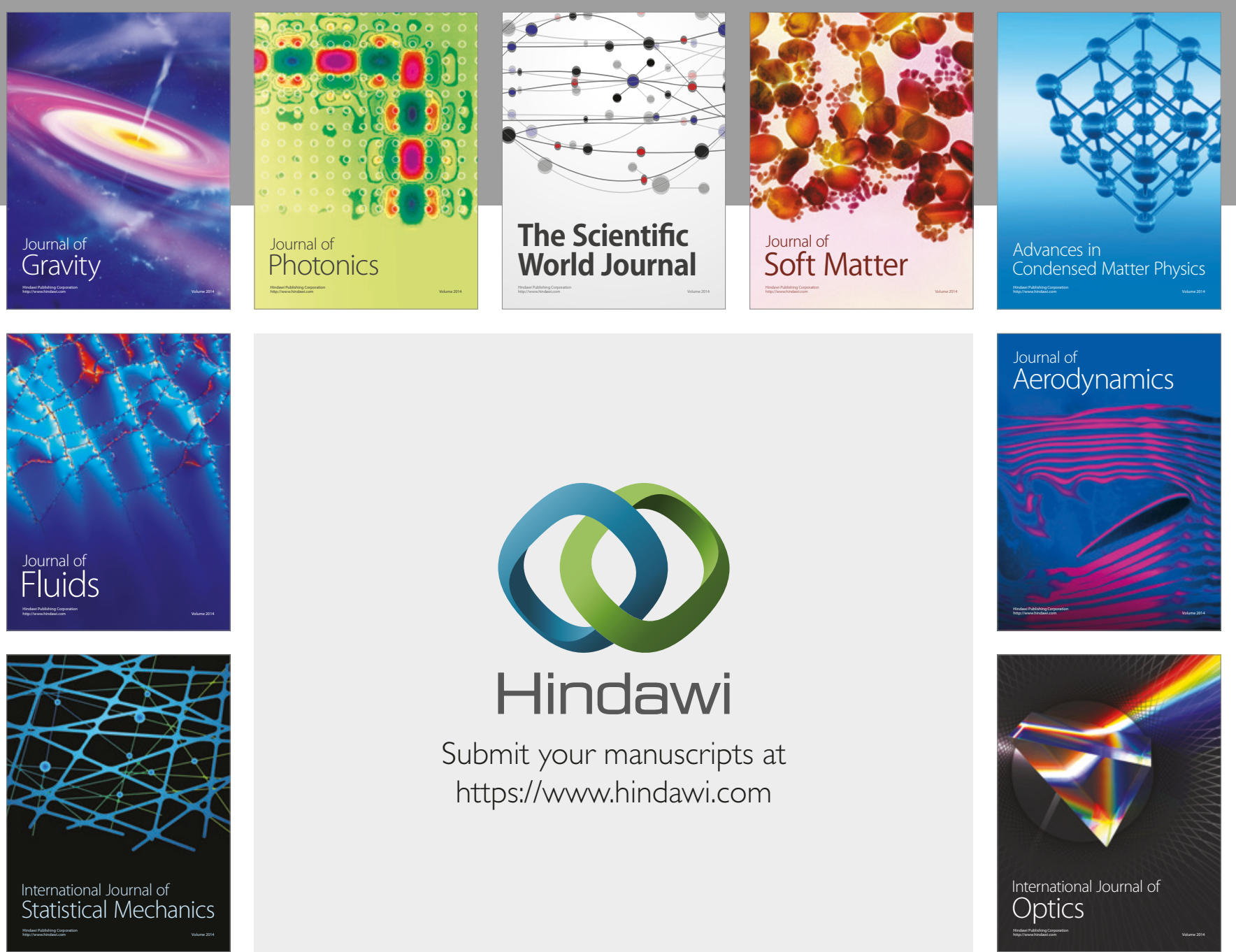

Submit your manuscripts at

https://www.hindawi.com
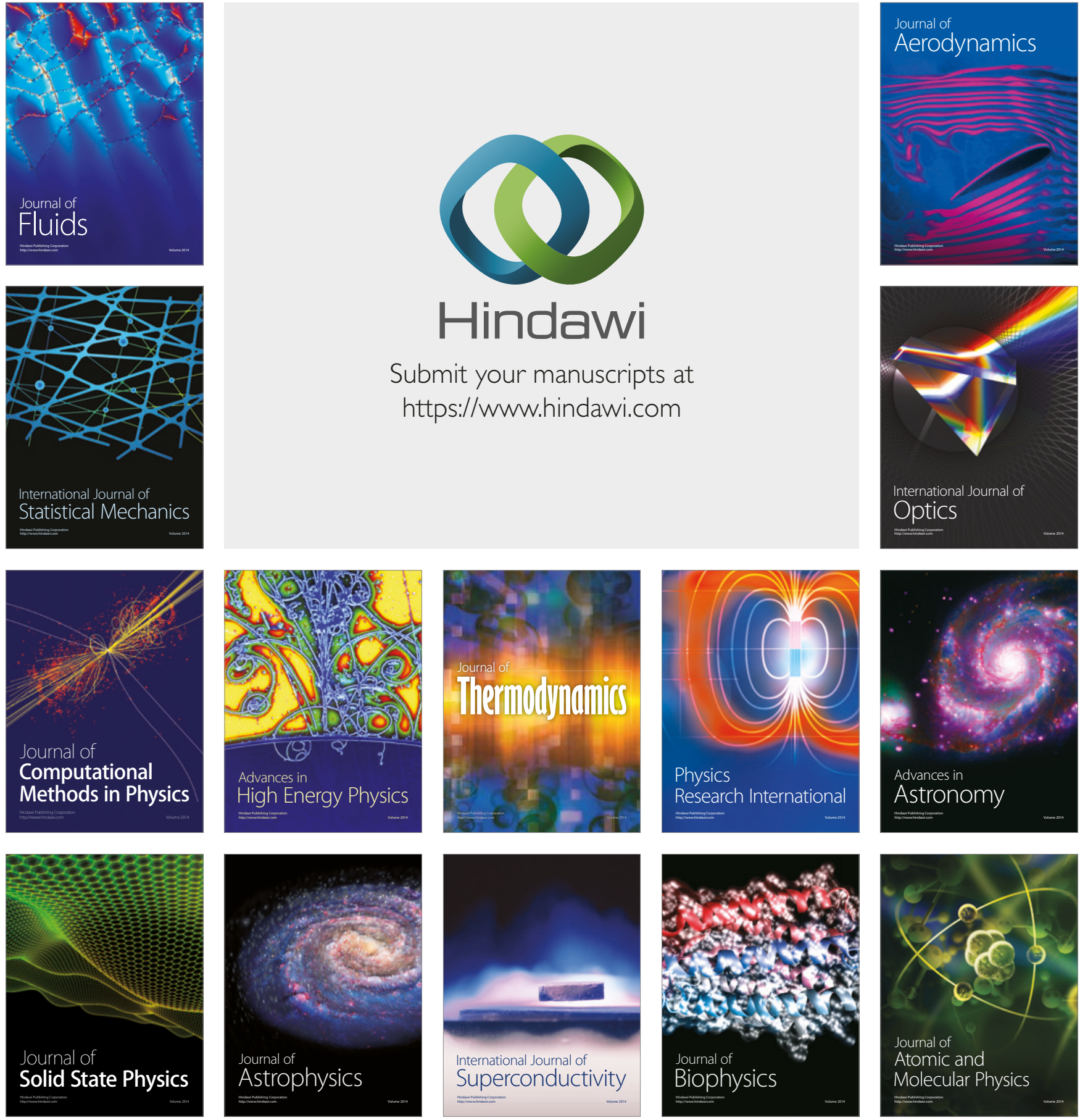